\title{
FNDC5/Irisin counteracts lipotoxic-induced apoptosis in hypoxic H9c2 cells
}

\author{
Isabel Moscoso1,2,*, María Cebro-Márquez1,*, Moisés Rodríguez-Mañero2,3, José Ramón González-Juanatey1,2,3 and \\ Ricardo Lage ${ }^{1,2}$
}

${ }^{1}$ Cardiology Group, Center for Research in Molecular Medicine and Chronic Diseases (CIMUS), Universidade de Santiago de Compostela and Health Research Institute, University Clinical Hospital of Santiago de Compostela, Santiago de Compostela, Spain ${ }^{2}$ Centro de Investigación Biomédica en Red de Enfermedades Cardiovasculares (CIBERCV), Madrid, Spain

${ }^{3}$ Department of Cardiology and Coronary Unit, University Clinical Hospital of Santiago de Compostela, Santiago de Compostela, Spain

Correspondence should be addressed to I Moscoso or R Lage: imosgal@gmail.com or rlagef@gmail.com

*(I Moscoso and M Cebro-Márquez contributed equally to this work)

\begin{abstract}
Irisin is a newly identified adipokine critical to modulate body metabolism, fatty acid metabolism and oxidative stress; recent evidence suggests a cardioprotective role in ischemic injury. Loss of cardiomyocytes during acute myocardial infarction is strongly associated with energetic changes and lipotoxic-induced apoptosis. Our aim was to study FNDC5/irisin's potential protective role against hypoxia and lipotoxicity, both related with myocardial infarction environment. H9c2 cells were treated with palmitate and/or irisin in normoxic/hypoxic conditions. Cell viability and apoptosis were assessed by MTT assay and annexin V/PI staining. Immunoblotting was used to confirm apoptotic cascade regulation. Irisin counteracts lipotoxic-induced apoptosis in hypoxic cardiomyoblasts by activating Akt signaling pathway suggesting the potential therapeutic role of irisin in ischemic heart disease.
\end{abstract}

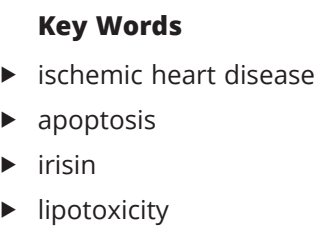

Journal of Molecular Endocrinology (2019) 63, 151-159

\section{Introduction}

Continuous cardiac contractile activity is a strictly controlled process that entails a high-energy demand to generate the necessary mechanical force but also to maintain cellular homeostasis. Energy demand is supported mainly through fatty acids (FAs) oxidation (Lopaschuk et al. 2010). Nevertheless, due to lack of myocardial oxygen supply, abrupt metabolic changes are produced after a coronary occlusion, compensatory adrenergic response and fatty acid overload turn FA metabolism deleterious (Geerling et al. 2014). Increased delivery, uptake or decreased FA turnover lead to myocardial lipid accumulation (Wende \& Abel 2010) and lipotoxicity (Wende \& Abel 2010). Myocardial lipotoxicity is associated to endoplasmic reticulum stress and mitochondrial dysfunction, altered calcium homeostasis, ceramide synthesis, oxidative stress, contractile dysfunction and apoptosis (Wende \& Abel 2010).

Irisin is a small polypeptide hormone cleaved from the fibronectin type III domain containing 5 (FNDC5) (Bostrom et al. 2012), is regulated by exercise and associated to healthy metabolic effects (Bostrom et al. 2012, Huh et al. 2012). Higher levels of FNDC5 expression were reported in brain, skeletal and cardiac muscle (Varela-Rodriguez et al. 2016). A growing body of evidence suggests a strong association between irisin and cardiovascular health (Lecker et al. 2012, Emanuele et al. 2014). Low levels of circulating irisin have been associated with unfavorable lipid profile (Oelmann et al. 2016), 
reduced aerobic performance (Lecker et al. 2012), increased risk of heart failure (HF) (Emanuele et al. 2014) and endothelial dysfunction (Lu et al. 2015). In addition to risk factors, recent studies establish a close relationship between irisin and cardiovascular disease (Lecker et al. 2012, Emanuele et al. 2014, Kuloglu et al. 2014, Xiang et al. 2014, Oelmann et al. 2016). Elderly that maintain elevated circulating irisin levels, despite the age-associated decline (Huh et al. 2012, 2014), showed lower rate of infarction (Emanuele et al. 2014), whereas unusually young infarcted individuals showed lower levels of irisin. Significantly low levels of irisin have been described in patients with coronary artery disease (CAD), which is negatively associated with the severity of artery disease (Deng 2016), as well as myocardial infarction (MI) (Kuloglu et al. 2014, Anastasilakis et al. 2017). Data from murine models showed that cardiac FNDC5 expression is regulated in infarcted animals (Kuloglu et al. 2014). Also, irisin attenuates ischemia/reperfusion (I/R)-associated cytotoxicity in rat cardiomyoblasts and that preconditioning prevents I/R-induced mitochondrial dysfunction, decreasing infarct size (Zhao et al. 2016, Wang et al. 2017, 2018). In addition, irisin overexpression leads to mitochondrial overdrive and increases reactive oxygen species (Ho et al. 2018). Based on previous studies were irisin levels are decreased in a lipotoxic context and its protective role against ischemic injury is also described, we hypothesized that irisin might directly protect cardiomyocyte against lipotoxic-induced apoptosis.

\section{Materials and methods}

\section{Cell culture and reagents}

H9c2 cells were obtained from ATCC. H9c2 cells were chosen because these cells maintain electrical and hormonal signal pathway elements of adult cardiac cells (Hescheler et al. 1991), in addition to sensitivity to hypoxia and energetic similarities to primary cardiomyocytes (Kuznetsov et al. 2015). H9c2 were cultured in $0.1 \%$ gelatin-coated plates with DMEM medium (Sigma-Aldrich) supplemented with $10 \%$ fetal bovine serum (FBS), antibiotics (100UI/mL penicillin, $100 \mu \mathrm{g} / \mathrm{mL}$ streptomycin) and $2 \mathrm{mM} \mathrm{L}$-glutamine, in a $5 \% \mathrm{CO}_{2}$ atmosphere at $37^{\circ} \mathrm{C}$. $\mathrm{H} 9 \mathrm{c} 2$ were seeded at least $24 \mathrm{~h}$ before treatments unless otherwise indicated and the experimental procedures were conducted upon reaching $80 \%$ confluence.

Cells were treated with palmitate (Sigma-Aldrich) to trigger in vitro lipotoxicity (Wende \& Abel 2010),
$5 \mathrm{nM}$ irisin (Sigma-Aldrich) and/or $20 \mu \mathrm{M}$ specific Akt inhibitor (10-NCP) (Merck). Palmitate was prepared by conjugation with fatty acid-free bovine serum albumin by dissolving in ethanol and diluting 100-fold in an aqueous $2 \%$ bovine serum albumin (BSA) solution to achieve selected concentration, and palmitate solution was freshly prepared for each experiment.

\section{Hypoxia induction}

H9c2 were cultured in a hypoxic chamber (Baker Ruskinn's Inviv $\mathrm{O}_{2} 200$, Bridgwater) under lower oxygen concentration $\left(0.1 \% \mathrm{O}_{2}, 5 \% \mathrm{CO}_{2}\right.$ and $\mathrm{N}_{2}$ balance) than oxygen demand for $24 \mathrm{~h}$ to mimic pathophysiologic ischemic environment (Lage et al. 2015).

\section{Cell viability and apoptosis}

Cell viability and apoptosis was measured using the MTT assay (Sigma-Aldrich) and FITC Annexin-V-FLUOS staining kit (Roche Diagnostics) according to the manufacturer's protocol. Briefly, H9c2 cells were seeded at a density of 5000 cells/well in 96-well plates. Cells were treated with $250 \mu \mathrm{M}$ or $500 \mu \mathrm{M}$ palmitate and/or $5 \mathrm{nM}$ irisin for $24 \mathrm{~h}$ under normoxic and hypoxic conditions. Later, MTT $(0.5 \mathrm{mg} / \mathrm{mL})$ was added and incubated at $37^{\circ} \mathrm{C}$ for $4 \mathrm{~h}$. Formazan crystals were solubilized with DMSO and isopropanol (1:1). The optical density (OD) was measured at a wavelength of $570 \mathrm{~nm}$ and $690 \mathrm{~nm}$ using an automated microplate reader. Otherwise, H9c2 cells were seeded at a density of $5 \times 10^{5}$ cells/well in sixwell plates; cells were then treated for $24 \mathrm{~h}$, collected by trypsinization and centrifuged at $300 \boldsymbol{g}$ for $5 \mathrm{~min}$ for flow cytometry. Following suspension in binding buffer, cells were labeled with Annexin-V-FITC and propidium iodide (PI) according to the manufacturer's instructions. FITC and PI were measured by flow cytometry analysis of 5000-gated cells using FACScan and CellQuestPro software from Becton Dickinson. Cell populations were defined as viable (AV-/PI-), apoptotic $(\mathrm{AV}+)$ or necrotic (PI+) cells.

\section{Real-time quantitative PCR}

RNA was isolated from cell culture or tissues using TRI Reagent ${ }^{\circledR}$ (Sigma-Aldrich). First-strand cDNA was synthesized from $1 \mu \mathrm{g}$ total RNA using RevertAid FirstStrand cDNA Synthesis kit (Thermo Scientific). The resulting cDNA was subjected to real-time PCR using FastStart Universal SYBR Green Master (Rox) (Roche Molecular 
Biochemicals) was performed using specific primers (FNDC5, Fw: GAGGTGCTGATCATCGTCGT, Rv: GAGCAAGCACTGAAAGGGTTT; 36B4, Fw: GGCG TCCTCATTAGAGTGACA, Rv: TAGTTGGACTTCCA GGTCGC). All reactions were carried out in an Eppendorf Realplex thermocycler. Sample values were standardized against an indicated housekeeping gene.

\section{Western blot}

H9c2 cells were homogenized in ice-cold lysis buffer containing $50 \mathrm{mmol} / \mathrm{L}$ Tris-HCl, pH 7.5, $1 \mathrm{mmol} / \mathrm{L}$ EGTA, $1 \mathrm{mmol} / \mathrm{L}$ EDTA, 1\% Triton X-100, $1 \mathrm{mmol} / \mathrm{L}$ sodium orthovanadate, $50 \mathrm{mmol} / \mathrm{L}$ sodium fluoride, $5 \mathrm{mmol} / \mathrm{L}$ sodium pyrophosphate, $0.27 \mathrm{~mol} / \mathrm{L}$ sucrose, $0.1 \%$ 2-mercaptoethanol and $1 \times$ Complete Protease Inhibitor Cocktail (Roche Diagnostics) for $1 \mathrm{~h}$. Homogenates were centrifuged at $21.100 \boldsymbol{g}$ for $15 \mathrm{~min}$ at $4^{\circ} \mathrm{C}$; supernatants were removed, and frozen at $-20^{\circ} \mathrm{C}$. Cells lysates $(15 \mu \mathrm{g})$ were subjected to $13 \%$ acrylamide SDS-PAGE gels, transferred on a PVDF membrane as previously described. Membranes were blocked for $1 \mathrm{~h}$ in TBS-Tween (TBST: $50 \mathrm{mmol} / \mathrm{L}$ Tris-HCl, pH 7.5, $0.15 \mathrm{~mol} / \mathrm{L} \mathrm{NaCl}$ and $0.1 \%$ Tween) containing 3\% BSA and probed with specific antibodies against Akt, phosphorylated Akt, Bax, phosphorylated Bad, Caspase-3 (Cell Signaling), FNDC5 (Abcam), B-actin (Sigma-Aldrich) and $\alpha$-tubulin (Santa Cruz). Detection of proteins was performed using horseradish peroxidase-conjugated secondary antibodies
Anti-Rabbit IgG (Cell Signaling) and Anti-Mouse IgG (Dako EnVision system, peroxidase) and an enhanced chemiluminescence reagent Pierce-ECL Western blotting substrate (Thermo Scientific).

Representative blots correspond to unedited images of full radiographic film detection. Each band is accompanied by its respective load control except for the ratio between cleaved and uncleaved Caspase 3 form. In fact, although Akt and phophoAkt was represented as a ratio, both load controls were included since samples should be incubated in individual membranes (for accuracy).

\section{Statistical analysis}

Data are represented as mean \pm s.E.M. (standard error of the media). Statistical significance was determined by MannWhitney test, ANOVA followed by Tukey's or Bonferroni's post hoc test or one-way ANOVA followed by Tukey's, post hoc test determined using GraphPad Prism 6 Software. $P<0.05$ was considered significant.

\section{Results}

\section{Decreased FNDC5 expression in hypoxic cardiomyoblasts}

H9c2 cells exposed to hypoxia showed a time-dependent decrease in FNDC5 mRNA and protein levels (Fig. 1A and B).
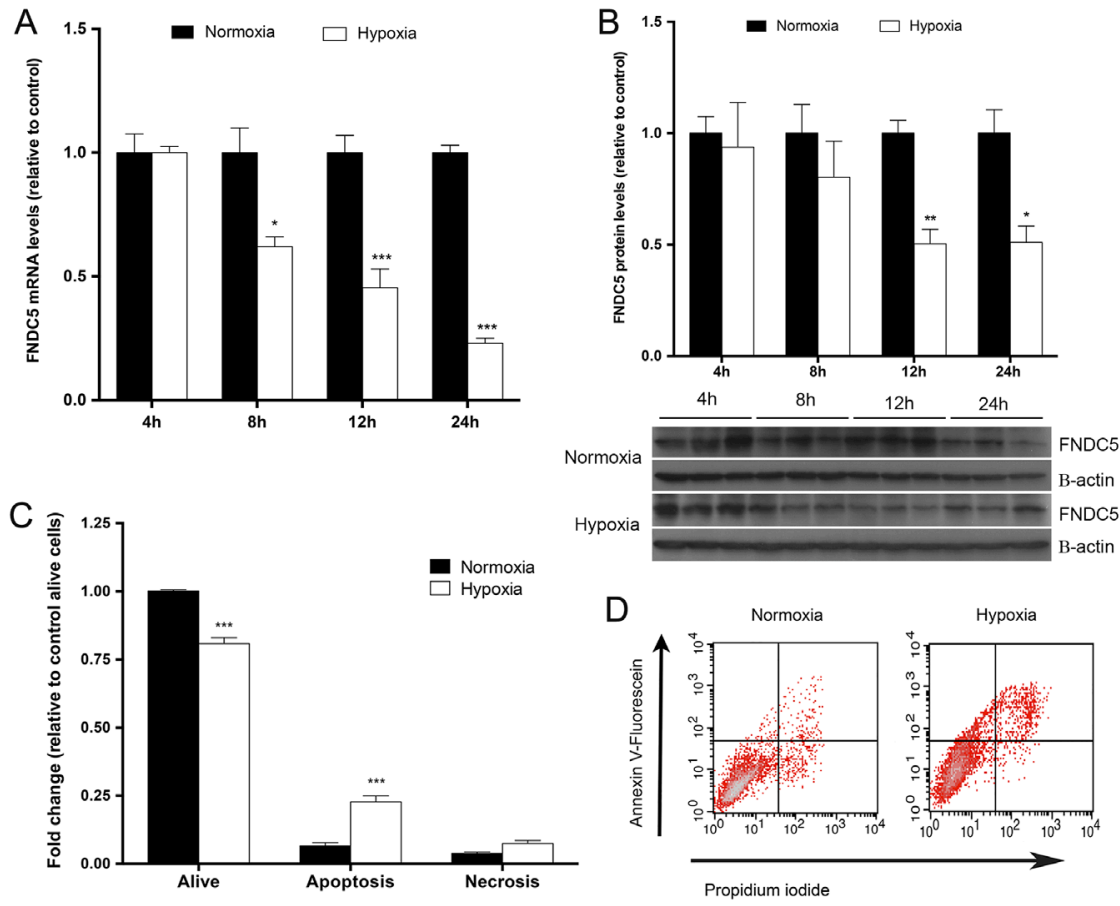

Figure 1

FNDC5 expression in $\mathrm{H} 9 \mathrm{c} 2$ cells under hypoxic conditions. (A) FNDC5 mRNA levels measured by real-time PCR in normoxic and hypoxic conditions. Data are expressed as mean \pm S.E.M. $(n=3), P$ value was determined by determined by two-way ANOVA followed by Bonferroni's post hoc test. (B) FNDC5 protein levels measured by Western blot in normoxic and hypoxic conditions. Data are expressed as mean \pm s.E.M. $(n=3), P$ value was determined by determined by two-way ANOVA followed by Bonferroni's post hoc test. (C) Annexin $\checkmark$ flow cytometry assay under normoxic and hypoxic conditions. $P$ value was determined by determined by two-way ANOVA followed by Bonferroni's post hoc test. (D) Representative density-plots images of Annexin V-FITC/propidium iodide double staining flow cytometry in normoxic and hypoxic conditions. Data are expressed as mean \pm S.E.M. $(n=4) . * P<0.05$ vs control; $* * P<0.01$ vs control; $* * \star P<0.001$ vs control. A full colour version of this figure is available at https://doi. org/10.1530/JME-19-0123. 
Flow cytometry study showed that lower expression of FNDC5 after $24 \mathrm{~h}$ of hypoxia is accompanied by a significant increase in $\mathrm{H} 9 \mathrm{c} 2$ cell apoptosis (Fig. 1C and D).

\section{Irisin prevents lipotoxic-induced apoptosis under hypoxic conditions}

Since lipotoxicity exacerbates cellular damage under ischemic conditions, we aimed to show if irisin supplementation exerts any protective effect against lipotoxic-induced apoptosis in hypoxic H9c2 cells. Despite the significant decrease in FDNC5 expression, our data failed to show a protective effect of irisin against hypoxia in the absence of lipotoxicity (data not shown). Also, our data showed that under normoxic conditions, irisin supplementation has no effect on viability, regardless of lipotoxicity (Fig. 2A). Nevertheless, our results clearly demonstrated that in hypoxic conditions, irisin supplementation improves cell viability $(0.39 \pm 0.04$ vs $0.63 \pm 0.04)$ and counteracts lipotoxic-induced apoptosis $(0.68 \pm 0.05$ vs $0.47 \pm 0.08)$ (Fig. $2 \mathrm{~B}$ and C).
Irisin prevents lipotoxic-induced increase of pro-apoptotic $\mathrm{Bcl}-2$ family members under hypoxic conditions

Pro-apoptotic Bcl-2 family protein expression was measured by Western blot analysis. As expected, lipotoxicity triggered a significant increase in Bax levels $(0.99 \pm 0.09$ vs $3.69 \pm 0.91)$ and Caspase- 3 activity (0.007 \pm 0.004 vs $0.99 \pm 0.205)$ as well as a significant decrease in phosphorylated Bad levels ( $1 \pm 0.16$ vs $0.12 \pm 0.02$ ). Our data showed that irisin supplementation prevents all the above-described lipotoxic-induced pro-apoptotic changes in Bax levels ( $3.69 \pm 0.91$ vs $1.53 \pm 0.18)$, Caspase- 3 activity $(0.99 \pm 0.205$ vs $0.31 \pm 0.01)$ and phosphorylated Bad levels $(0.12 \pm 0.02$ vs $0.31 \pm 0.09)$ (Fig. $3 \mathrm{~A}$ and $\mathrm{B})$.

\section{Akt mediates irisin cardioprotective effects against lipotoxicity}

Irisin supplementation stimulated a significant increase of pAkt/Akt ratio in palmitate-treated group

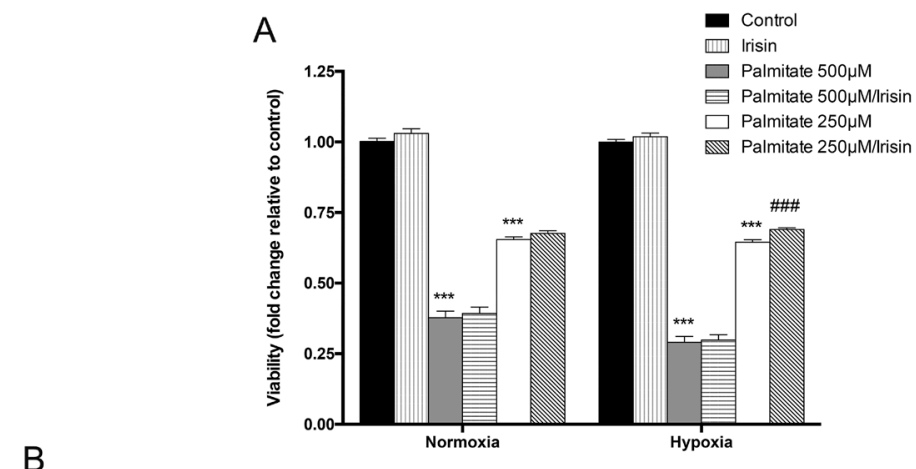

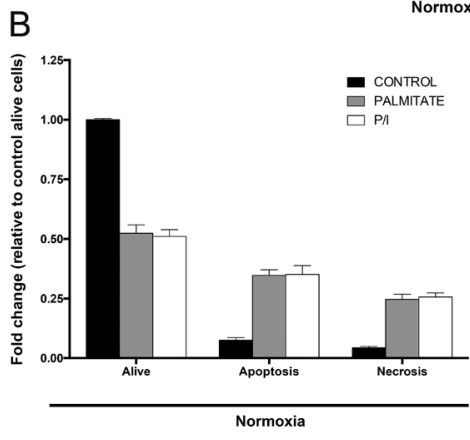

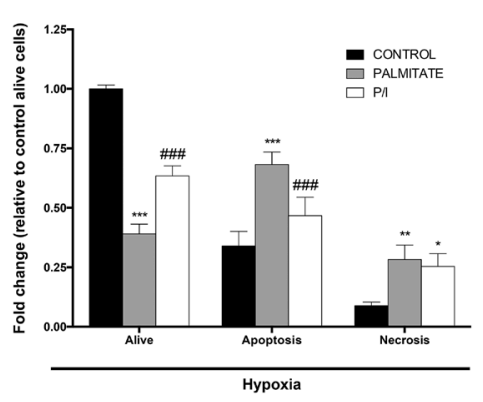

C
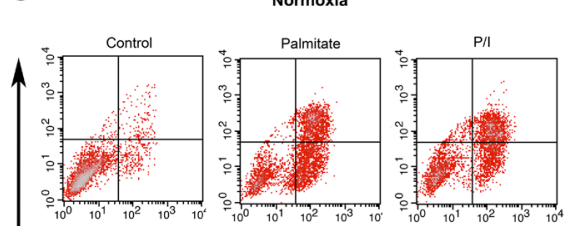

Hypoxia

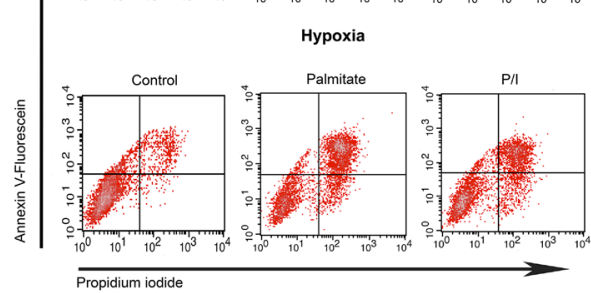
https://doi.org/10.1530/JME-19-0123
(C) 2019 Society for Endocrinology Published by Bioscientifica Ltd. Printed in Great Britain

\section{Figure 2}

Irisin prevents lipotoxic-induced H9c2 cells apoptosis under hypoxic conditions. (A) MTT cell viability assay in $\mathrm{H} 9 \mathrm{c} 2$ cells treated with palmitate in normoxic and hypoxic conditions $(n=4)$. (B) Annexin $V$ flow cytometry assay of lipotoxicinduced apoptosis under normoxic and hypoxic conditions. Histogram represents the viable, apoptotic and necrotic rate of $\mathrm{H} 9 \mathrm{c} 2$ cells relative to control group. Data are expressed as mean \pm S.E.M. $(n=4)$. (C) Representative density plots images of Annexin V-FITC/propidium iodide double staining flow cytometry in normoxic and hypoxic conditions. ${ }^{*} P<0.05$ vs control; ${ }^{*} P<<0.01$ and $* * * P<0.001 ; \# \# P<0.001$ vs palmitate. $P$ values determined by two-way ANOVA followed by Tukey's post hoc test. A full colour version of this figure is available at https://doi.org/10.1530/ JME-19-0123. 


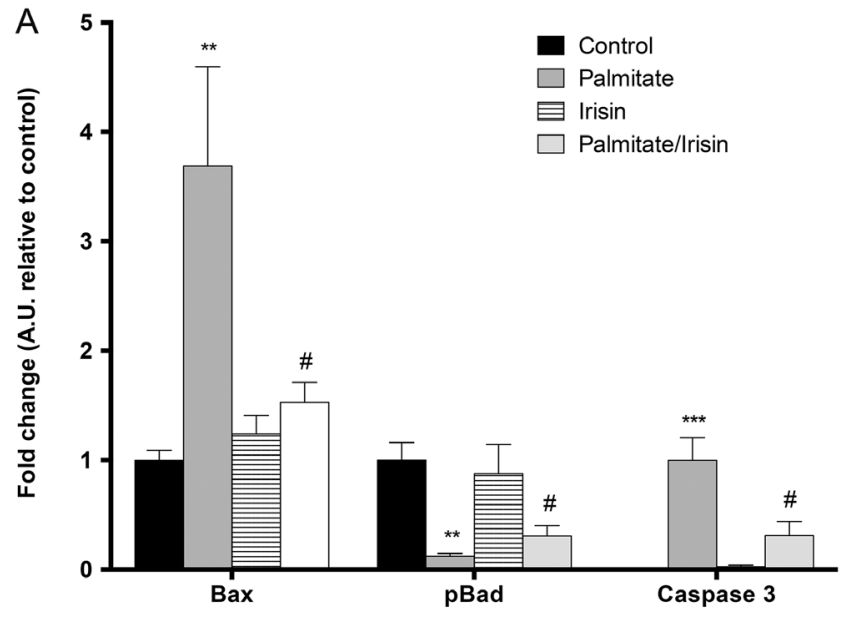

B

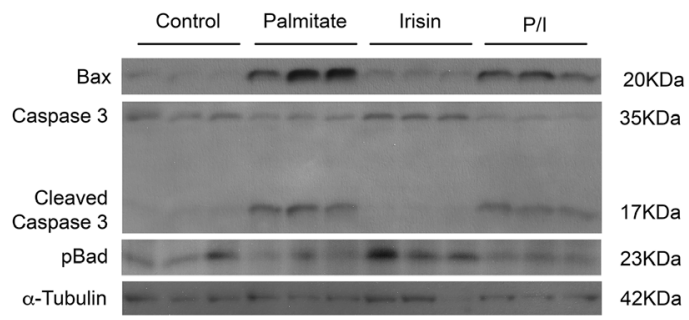

\section{Figure 3}

Caspase-3 and $\mathrm{BCl}-2$ family proteins expression in irisin and/or palmitate H9c2-treated cells under hypoxic conditions. (A) Histogram represents mean \pm S.E.M. relative to control group $(n=3)$. Caspase-3 is represented as cleaved to full-length form. (B) Representative Western blots are shown for $\mathrm{H} 9 \mathrm{c} 2$ cells exposed to palmitate/irisin. White spaces indicate splicing of cropped lines from different parts of the same gel or from different gels. $\alpha$-Tubulin is shown as a loading control. Band intensity was quantified regarding Caspase 3 or $\alpha$-Tubulin expression. $* * P<0.01$ vs control; $* \star * P<0.001$ vs control and $\# P<0.05$ vs palmitate. $P$ values determined by one-way ANOVA followed by Tukey's post hoc test.

(14.24 \pm 6.26 vs $45.67 \pm 16.51$ ) (Fig. 4 A and B). To determine if increased Akt activity mediates cardioprotective role of irisin, annexin $\mathrm{V}$ staining flow cytometry assay was performed in palmitate/irisin treated cells co-treated with 10-NCP (Fig. 4C and D). Akt inhibition counteracts antiapoptotic irisin effects (P: $0.50 \pm 0.04$ vs P/I: $0.38 \pm 0.04$ vs P/I/10-NCP:0.54 \pm 0.06$)$.

\section{Discussion}

As it is widely accepted that maintaining regular physical activity reduces the risk of cardiovascular events, it is also known that exercise-based cardiac rehabilitation improves myocardial remodeling, recurrence and all-cause mortality (Suaya et al. 2009, Hammill et al. 2010, Goel et al. 2011, Lawler et al. 2011). Boström et al. identified a new potential molecular mechanism by which physical activity improves overall metabolism by increasing irisin levels.
Beyond the general metabolic improvement, cumulative evidence to date established a strong relationship between irisin and cardiovascular health. Curiously, there seems to be a threshold in both cases, as well as excessive exercise after MI has been related with adverse cardiac remodeling and scar formation (Kloner \& Kloner 1983), excessive irisin levels have been related with increased oxidative stress and cardiomyocyte apoptosis (Ho et al. 2018). These observations are in accordance with recent clinical results, which conclude that elevated serum concentrations of irisin are associated with the development of major adverse cardiovascular events in patients with established CAD after revascularization (Aronis et al. 2015) and also related with adverse cardiovascular outcomes in both acute heart failure (Shen et al. 2017) and infarcted patients (Hsieh et al. 2018). Although the relationship and benefit of irisin on the myocardium is demonstrated, there remains some controversy mainly based on the experimental approaches (inadequate doses, short experimental groups, clinical confounding variables) and technical limitations such as antibody specificity and poor accuracy of ELISA kits (Jedrychowski et al. 2015), but also neither it can be ruled out that irisin may have the double-edged effect.

Our data placed the focus on the role that irisin might play against lipotoxic injury associated to ischemic events. Recent study of the FNDC5 gene expression profile in different tissues in adult rats showed that cardiac muscle is one of the tissues with the highest expression of irisin (Varela-Rodriguez et al. 2016). Our data demonstrated for the first time that FNDC5 expression, both mRNA and protein, is downregulated in a time-dependent manner in hypoxic H9c2 cells. Also, our data showed that lower levels of irisin observed after $24 \mathrm{~h}$ of hypoxia are accompanied to increased apoptosis. Decreased cardiomyocyte irisin expression has been previously described in murine model of MI (Kuloglu et al. 2014). Suppression of uncoupled activity of irisin in an energy-depleting context such as lack of oxygen sounds coherent. Curiously, in the MI murine model, it was found that irisin serum expression is decreased but it is accompanied with an increase in the surrounding connective tissue amount in which irisin expression is increased, suggesting that the maintenance of physiological irisin concentrations around cardiac muscle might be important during MI. Reduction of in vitro reperfusion injury (Zhao et al. 2016) and infarct size in in vivo models, together with the potential existence of an irisin-specific receptor (Xie et al. 2015) strongly suggest that myocardium might respond to circulating irisin levels and that there are cardiovascular benefits beyond energy homeostasis. 
A
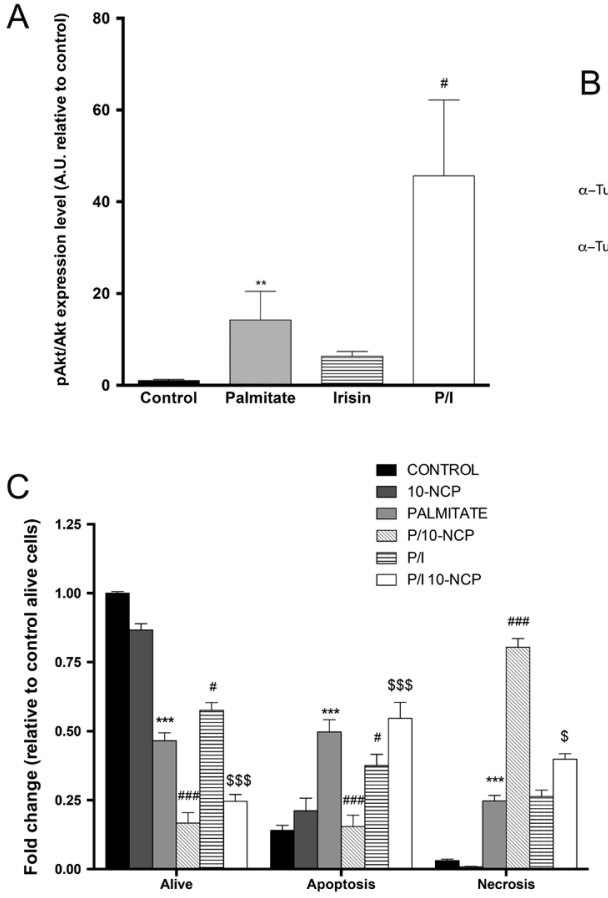

D

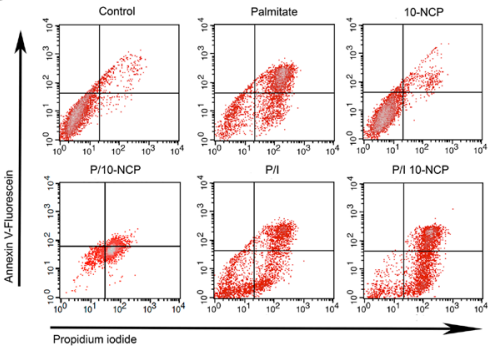

Figure 4

Akt inhibition counteracts irisin effects in lipotoxic-induced $\mathrm{H} 9 \mathrm{c} 2$ cells apoptosis under hypoxic conditions. (A) Histogram represents mean \pm S.E.M. relative to control group $(n=3)$. Akt is represented as phosphorylated to nonphosphorylated form. $P$ values determined by one-way ANOVA followed by Tukey's post hoc test. (B) Representative Western blots are shown for H9c2 cells exposed to palmitate/irisin. White spaces indicate splicing of cropped lines from different parts of the same gel or from different gels. $\alpha$-Tubulin is shown as a loading control. Band intensity was quantified regarding $\alpha$-Tubulin expression. (C) Annexin $\vee$ flow cytometry assay of $\mathrm{H} 9 \mathrm{c} 2$ cells treated with palmitate, irisin and/or 10-NCP under hypoxic conditions. Histogram represents the viable, apoptotic and necrotic rate of H9c2 cells relative to control group. Data are expressed as mean \pm S.E.M. $(n=4)$. $P$ values determined by two-way ANOVA followed by Tukey's post hoc test. (D) Representative density-plot images of Annexin V-FITC/propidium iodide double staining flow cytometry. $\star \star \star * P<0.001$ vs control; $\# P<0.05$ vs palmitate; $\# \# P<0.001$ vs palmitate; $\$ P<0.05$ and $\$ \$ P<0.001$ vs palmitate/irisin. A full colour version of this figure is available at https://doi.org/10.1530/ JME-19-0123.
Sudden myocardial metabolic changes, due to lack of oxygen supply and compensatory adrenergic response (Geerling et al. 2014) finally trigger a lipotoxic environment (Wende \& Abel 2010), central in MI cardiomyocyte apoptosis. In addition to previously described benefits in reperfusion injury, our data show a protective role of irisin in this context.

Previousstudiesshowed thatirisininduces proliferation in endothelial (Song et al. 2014) and neuronal (Moon et al. 2013) cell lines. However, increased proliferative activity was described at pharmacological but not physiological concentrations (Moon et al. 2013). According to previous studies (Xie et al. 2015), our data demonstrated that irisin supplementation failed to increase cell proliferation at physiological concentrations ( $5 \mathrm{nM}$ ) (data not shown) but also, flow cytometry analysis confirmed that the observed increased viability is associated with the prevention of lipotoxic-induced apoptosis in hypoxia. Anti-apoptotic activity of irisin was also confirmed through immunoblot studies that evidenced the regulation of apoptotic pathways. Increased phosphorylation of pro-apoptotic Bcl-2 family protein Bad (inactive form) accompanied by a drop in Bax and Caspase-3 expression. Since Akt is involved in a wide range of cellular processes including apoptosis and regulation of myocardial metabolism by irisin (Xie et al. 2015), our data showed that selective pharmacological Akt inhibition counteracted the anti-apoptotic role of irisin, demonstrating that activation of Akt signaling pathway is implicated in protective role of irisin.

One of the highlights of this study is that irisin expression and function seems to be related to pathophysiological environment. Protective role of irisin against lipotoxic injury, only in hypoxic conditions, may be related to metabolic shift in conditions of deficient oxygen supply (Lopaschuk et al. 2010) and metabolic effects of irisin depend on substrate availability (Vaughan et al. 2015). Pathophysiological response to acute MI is associated with a sudden increase in circulating FA simultaneously to increased FA translocation and intramyocardial lipid accumulation (Lopaschuk et al. 2010) that contributes to cardiomyocyte apoptosis (Wende \& Abel 2010). For that reason, it is essential to address and counteract the deleterious effects of FA overload, especially in ischemic conditions. Our data indicate that irisin counteracts lipotoxic-induced injury and suggest that differential FNDC5 expression might occur as a coordinated response or as a consequence of the context of lack of oxygen and lipid overload to modulate fatty acid oxidation. In this sense, decreased myocardial expression of FNDC5 might reduce fatty acid oxidation to avoid oxygen consumption beyond its possibilities and oxidative stress; more studies are required to assess this possibility. 

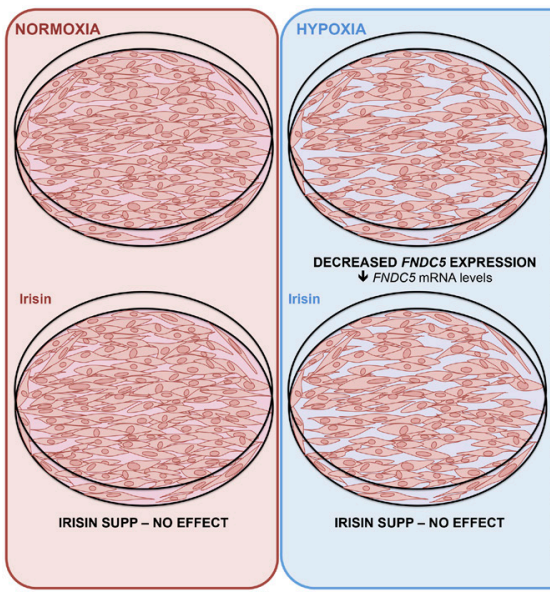

Undoubtedly, there are certain considerations to take into account in our study. Cardiomyoblasts cell line could not be compared with animal models or primary cultures. However, we aimed for this model because it allows us to work with an energetically differentiated myocardial cell line, closest to adult cardiomyocytes and avoid external conditioning of in vivo models. Another issue to highlight is that contrary to previous data (Zhao et al. 2016), our data failed to show any cardioprotective role in hypoxic conditions, in the absence of lipotoxicity. In this sense, previous positive studies were carried out considering reperfusion injury but also using pharmacological concentrations of irisin (Zhao et al. 2016), while in our model irisin concentration is closer to physiological concentrations. This might be the reason why prevention of damage is not effective in this model; however, our data are consistent with the previously described increase of irisin expression in surrounding connective tissue since increased local levels of irisin might be enough to reduce cardiomyocyte apoptosis during MI. New studies that locally overexpress FNDC5, near to the ischemic region, in murine models of infarction, are needed to solve this question. Another outstanding question is that we did not observe changes in irisin effects in normoxia, unlike in a hypoxic environment. In this sense, it seems that irisin might play a different role depending on the pathophysiological state.

Despite experimental and technical limitations, a huge number of studies showed that irisin plays a central role in cardiovascular health and key issues remain unresolved by the scientific community. Literature, together with the data obtained in this study, clearly demonstrate that myocardial expression of FNDC5 is regulated in pathophysiological conditions, showing a direct protective role of irisin against lipotoxicity in addition to previously described reperfusion injury, but also strongly suggest that local increase, closest to infarcted area, might be an adaptive response to reduce ischemic damage (Fig. 5).

\section{Conclusion}

Our in vitro model mimicking a lipotoxic context characteristic of hyperlipidemic states in ischemic events shows that irisin counteracts lipotoxic-induced apoptosis in hypoxic cardiomyoblast in an Akt-dependent manner. Undoubtedly, future in-depth studies in animal models of acute MI are needed to address the mechanism as well as functional significance of differential FNDC5 expression under pathophysiological conditions.

\section{Declaration of interest}

The authors declare that there is no conflict of interest that could be perceived as prejudicing the impartiality of the research reported.

\section{Funding}

This work was supported by Red de Investigación Cardiovascular (RIC) (RD12/0042/0039) an initiative of ISCIII, Programa de Consolidación de Unidades de Investigación Competitivas do SUG (GPC 2013-051) of Xunta de Galicia.

\section{References}

Anastasilakis AD, Koulaxis D, Kefala N, Polyzos SA, Upadhyay J, Pagkalidou E, Economou F, Anastasilakis CD \& Mantzoros CS 2017 Circulating irisin levels are lower in patients with either stable coronary artery disease (CAD) or myocardial infarction (MI) versus healthy controls, whereas follistatin and activin A levels are higher and can discriminate MI from CAD with similar to CK-MB accuracy. Metabolism: Clinical and Experimental 73 1-8. (https://doi. org/10.1016/j.metabol.2017.05.002) 
Aronis KN, Moreno M, Polyzos SA, Moreno-Navarrete JM, Ricart W, Delgado E, De La Hera J, Sahin-Efe A, Chamberland JP, Berman R, et al. 2015 Circulating irisin levels and coronary heart disease: association with future acute coronary syndrome and major adverse cardiovascular events. International Journal of Obesity 39 156-161. (https://doi.org/10.1038/ijo.2014.101)

Bostrom P, Wu J, Jedrychowski MP, Korde A, Ye L, Lo JC, Rasbach KA, Bostrom EA, Choi JH, Long JZ, et al. 2012 A PGC1-alpha-dependent myokine that drives brown-fat-like development of white fat and thermogenesis. Nature 481 463-468. (https://doi.org/10.1038/ nature10777)

Deng W 2016 Association of serum irisin concentrations with presence and severity of coronary artery disease. Medical Science Monitor 22 4193-4197. (https://doi.org/10.12659/msm.897376)

Emanuele E, Minoretti P, Pareja-Galeano H, Sanchis-Gomar F, Garatachea N \& Lucia A 2014 Serum irisin levels, precocious myocardial infarction, and healthy exceptional longevity. American Journal of Medicine 127 888-890. (https://doi.org/10.1016/j. amjmed.2014.04.025)

Geerling JJ, Boon MR, Kooijman S, Parlevliet ET, Havekes LM, Romijn JA, Meurs IM \& Rensen PC 2014 Sympathetic nervous system control of triglyceride metabolism: novel concepts derived from recent studies. Journal of Lipid Research 55 180-189. (https:// doi.org/10.1194/jlr.R045013)

Goel K, Lennon RJ, Tilbury RT, Squires RW \& Thomas RJ 2011 Impact of cardiac rehabilitation on mortality and cardiovascular events after percutaneous coronary intervention in the community. Circulation 123 2344-2352. (https://doi.org/10.1161/ CIRCULATIONAHA.110.983536)

Hammill BG, Curtis LH, Schulman KA \& Whellan DJ 2010 Relationship between cardiac rehabilitation and long-term risks of death and myocardial infarction among elderly medicare beneficiaries. Circulation 121 63-70. (https://doi.org/10.1161/ CIRCULATIONAHA.109.876383)

Hescheler J, Meyer R, Plant S, Krautwurst D, Rosenthal W \& Schultz G 1991 Morphological, biochemical, and electrophysiological characterization of a clonal cell $(\mathrm{H} 9 \mathrm{c} 2)$ line from rat heart. Circulation Research 69 1476-1486. (https://doi.org/10.1161/01. res.69.6.1476)

Ho MY, Wen MS, Yeh JK, Hsieh IC, Chen CC, Hsieh MJ, Tsai ML, Yang CH, Wu VC, Hung KC, et al. 2018 Excessive irisin increases oxidative stress and apoptosis in murine heart. Biochemical and Biophysical Research Communications 503 2493-2498. (https://doi. org/10.1016/j.bbrc.2018.07.005)

Hsieh IC, Ho MY, Wen MS, Chen CC, Hsieh MJ, Lin CP, Yeh JK, Tsai ML, Yang CH, Wu VC, et al. 2018 Serum irisin levels are associated with adverse cardiovascular outcomes in patients with acute myocardial infarction. International Journal of Cardiology $\mathbf{2 6 1}$ 12-17. (https://doi.org/10.1016/j.ijcard.2017.11.072)

Huh JY, Panagiotou G, Mougios V, Brinkoetter M, Vamvini MT, Schneider BE \& Mantzoros CS 2012 FNDC5 and irisin in humans: I. Predictors of circulating concentrations in serum and plasma and II mRNA expression and circulating concentrations in response to weight loss and exercise. Metabolism: Clinical and Experimental $6 \mathbf{1}$ 1725-1738. (https://doi.org/10.1016/j.metabol.2012.09.002)

Huh JY, Mougios V, Kabasakalis A, Fatouros I, Siopi A, Douroudos II, Filippaios A, Panagiotou G, Park KH \& Mantzoros CS 2014. Exerciseinduced irisin secretion is independent of age or fitness level and increased irisin may directly modulate muscle metabolism through AMPK activation. Journal of Clinical Endocrinology and Metabolism 99 E2154-E2161. (https://doi.org/10.1210/jc.2014-1437)

Jedrychowski MP, Wrann CD, Paulo JA, Gerber KK, Szpyt J, Robinson MM, Nair KS, Gygi SP \& Spiegelman BM 2015 Detection and quantitation of circulating human irisin by tandem mass spectrometry. Cell Metabolism 22 734-740. (https://doi.org/10.1016/j. cmet.2015.08.001)
Kloner RA \& Kloner JA 1983 The effect of early exercise on myocardial infarct scar formation. American Heart Journal 106 1009-1013. (https://doi.org/10.1016/0002-8703(83)90645-2)

Kuloglu T, Aydin S, Eren MN, Yilmaz M, Sahin I, Kalayci M, Sarman E, Kaya N, Yilmaz OF, Turk A, et al. 2014 Irisin: a potentially candidate marker for myocardial infarction. Peptides 55 85-91. (https://doi. org/10.1016/j.peptides.2014.02.008)

Kuznetsov AV, Javadov S, Sickinger S, Frotschnig S \& Grimm M 2015. H9c2 and HL-1 cells demonstrate distinct features of energy metabolism, mitochondrial function and sensitivity to hypoxiareoxygenation. Biochimica and Biophysica Acta 1853 276-284. (https://doi.org/10.1016/j.bbamcr.2014.11.015)

Lage R, Moscoso I, Fernandez-Trasancos Á, Cebro M, Couselo M, Fandino-Vaquero R, Bravo SB, Sierra J, Gonzalez-Juanatey JR \& Eiras S 2015 Differential behaviour of epicardial adipose tissuesecretomes with high and low orosomucoid levels from patients with cardiovascular disease in H9C2 cells. Molecular and Cellular Endocrinology 416 77-87. (https://doi.org/10.1016/j.mce.2015.08.025)

Lawler PR, Filion KB \& Eisenberg MJ 2011 Efficacy of exercise-based cardiac rehabilitation post-myocardial infarction: a systematic review and meta-analysis of randomized controlled trials. American Heart Journal 162 571.e2-584.e2. (https://doi.org/10.1016/j. ahj.2011.07.017)

Lecker SH, Zavin A, Cao P, Arena R, Allsup K, Daniels KM, Joseph J, Schulze PC \& Forman DE 2012 Expression of the irisin precursor FNDC5 in skeletal muscle correlates with aerobic exercise performance in patients with heart failure. Circulation: Heart Failure 5 812-818. (https://doi.org/10.1161/ CIRCHEARTFAILURE.112.969543)

Lopaschuk GD, Ussher JR, Folmes CD, Jaswal JS \& Stanley WC 2010 Myocardial fatty acid metabolism in health and disease. Physiological Reviews 90 207-258. (https://doi.org/10.1152/physrev.00015.2009)

Lu J, Xiang G, Liu M, Mei W, Xiang L \& Dong J 2015 Irisin protects against endothelial injury and ameliorates atherosclerosis in apolipoprotein E-Null diabetic mice. Atherosclerosis 243 438-448. (https://doi.org/10.1016/j.atherosclerosis.2015.10.020)

Moon HS, Dincer F \& Mantzoros CS 2013 Pharmacological concentrations of irisin increase cell proliferation without influencing markers of neurite outgrowth and synaptogenesis in mouse H19-7 hippocampal cell lines. Metabolism: Clinical and Experimental 62 1131-1136. (https://doi.org/10.1016/j. metabol.2013.04.007)

Oelmann S, Nauck M, Volzke H, Bahls M \& Friedrich N 2016 Circulating irisin concentrations are associated with a favourable lipid profile in the general population. PLOS ONE 11 e0154319. (https://doi. org/10.1371/journal.pone.0154319)

Shen S, Gao R, Bei Y, Li J, Zhang H, Zhou Y, Yao W, Xu D, Zhou F, Jin M, et al. 2017 Serum irisin predicts mortality risk in acute heart failure patients. Cellular Physiology and Biochemistry 42 615-622. (https://doi.org/10.1159/000477867)

Song H, Wu F, Zhang Y, Zhang Y, Wang F, Jiang M, Wang Z, Zhang M, Li S, Yang L, et al. 2014 Irisin promotes human umbilical vein endothelial cell proliferation through the ERK signaling pathway and partly suppresses high glucose-induced apoptosis. PLOS ONE 9 e110273. (https://doi.org/10.1371/journal.pone.0110273)

Suaya JA, Stason WB, Ades PA, Normand SL \& Shepard DS 2009 Cardiac rehabilitation and survival in older coronary patients. Journal of the American College of Cardiology 54 25-33. (https://doi.org/10.1016/j. jacc.2009.01.078)

Varela-Rodriguez BM, Pena-Bello L, Juiz-Valina P, Vidal-Bretal B, Cordido F \& Sangiao-Alvarellos S 2016 FNDC5 expression and circulating irisin levels are modified by diet and hormonal conditions in hypothalamus, adipose tissue and muscle. Scientific Reports 6 29898. (https://doi.org/10.1038/srep29898)

Vaughan RA, Gannon NP, Mermier CM \& Conn CA 2015 Irisin, a unique non-inflammatory myokine in stimulating skeletal muscle 
metabolism. Journal of Physiology and Biochemistry 71 679-689. (https://doi.org/10.1007/s13105-015-0433-9)

Wang H, Zhao YT, Zhang S, Dubielecka PM, Du J, Yano N, Chin YE, Zhuang S, Qin G \& Zhao TC 2017 Irisin plays a pivotal role to protect the heart against ischemia and reperfusion injury. Journal of Cellular Physiology 232 3775-3785. (https://doi.org/10.1002/jcp.25857)

Wang Z, Chen K, Han Y, Zhu H, Zhou X, Tan T, Zeng J, Zhang J, Liu Y, Li Y, et al. 2018 Irisin protects heart against ischemia-reperfusion injury through a SOD2-dependent mitochondria mechanism. Journal of Cardiovascular Pharmacology 72 259-269. (https://doi.org/10.1097/ FJC.0000000000000608)

Wende AR \& Abel ED 2010 Lipotoxicity in the heart. Biochimica and Biophysica Acta 1801 311-319. (https://doi.org/10.1016/j. bbalip.2009.09.023)
Xiang L, Xiang G, Yue L, Zhang J \& Zhao L 2014 Circulating irisin levels are positively associated with endothelium-dependent vasodilation in newly diagnosed type 2 diabetic patients without clinical angiopathy. Atherosclerosis 235 328-333. (https://doi.org/10.1016/j. atherosclerosis.2014.04.036)

Xie C, Zhang Y, Tran TD, Wang H, Li S, George EV, Zhuang H, Zhang P, Kandel A, Lai Y, et al. 2015 Irisin controls growth, intracellular $\mathrm{Ca} 2+$ signals, and mitochondrial thermogenesis in cardiomyoblasts. PLOS ONE 10 e0136816. (https://doi.org/10.1371/ journal.pone.0136816)

Zhao YT, Wang H, Zhang S, Du J, Zhuang S \& Zhao TC 2016 Irisin ameliorates hypoxia/reoxygenation-induced injury through modulation of histone deacetylase 4. PLOS ONE 11 e0166182. (https://doi.org/10.1371/journal.pone.0166182)

Received in final form 11 June 2019

Accepted 8 July 2019

Accepted Preprint published online 8 July 2019
(C) 2019 Society for Endocrinology Published by Bioscientifica Ltd. Printed in Great Britain 